\title{
The dual EGF/VEGF receptor tyrosine kinase inhibitor AEE788 inhibits growth of human hepatocellular carcinoma xenografts in nude mice
}

\author{
KINYA OKAMOTO $^{1,2^{*}}$, DANIEL NEUREITER $^{3 *}$, BEATE ALINGER $^{3}$, MATTHIAS MEISSNITZER $^{3}$, \\ GABRIELE SASS $^{4}$, VOLKER SCHMITZ ${ }^{5}$, PIETRO DI FAZIO ${ }^{1,8}$, TILL WISSNIOWSKI ${ }^{1}$, \\ SUSANNE GAHR $^{1}$, BERND HOHENSTEIN ${ }^{6}$, BERNHARD KAUFMANN ${ }^{7}$, AXEL SCHLÖSSER $^{7}$, \\ ULRIKE HAUS $^{7}$, ECKHART G. HAHN ${ }^{1}$, CHRISTOPH HEROLD ${ }^{1}$ and MATTHIAS OCKER ${ }^{1}$
}

\begin{abstract}
${ }^{1}$ Department of Medicine 1, University Hospital Erlangen, Erlangen, Germany; ${ }^{2}$ Second Department of Internal Medicine, Tottori University School of Medicine, Tottori, Japan; ${ }^{3}$ Institute of Pathology, Salzburger Landeskliniken, Paracelsus Private Medical University, Salzburg, Austria; ${ }^{4}$ Division of Experimental Immunology and Hepatology, Center of Internal Medicine, University Medical Centre Hamburg-Eppendorf, Hamburg; ${ }^{5}$ Department of Internal Medicine I, University Hospital Bonn, Bonn; ${ }^{6}$ Department of Medicine 4, University Hospital Erlangen, Erlangen; ${ }^{7}$ Novartis Pharma GmbH, Nuremberg, Germany; ${ }^{8}$ Dipartimento di Scienze Biochimiche, Università de Palermo, Policlinico, Palermo, Italy
\end{abstract}

Received April 29, 2008; Accepted July 11, 2008

DOI: 10.3892/ijo_00000059

\begin{abstract}
We investigated the effect of AEE788, a novel dual receptor tyrosine kinase inhibitor of the EGF and the VEGF receptor, for treatment of human HCC cell lines and in a subcutaneous xenograft model. Cell viability and apoptosis of HepG2 and Hep3B cells incubated with 0.1-100 $\mu \mathrm{M}$ AEE788 were quantified. In vivo, HepG2 cells were xenografted to NMRI mice and animals were treated orally with $50 \mathrm{mg} / \mathrm{kg}$ AEE788 3x/week. Immunohistochemistry and quantitative Western blotting was performed for pathway analysis in vitro and in vivo. AEE788 reduced growth and induced apoptosis of HCC cells by disrupting mitochondrial transmembrane potentials and inhibiting MAPK phosphorylation. In the xenografts, AEE788 lead to a reduced tumor growth by reducing proliferation and vascularisation. Except for a reversible skin reaction and weight loss, no signs of toxicity were observed. AEE788 is a promising new option for the treatment of HCC.
\end{abstract}

\section{Introduction}

Hepatocellular carcinoma (HCC) is the fifth most common malignancy in the world and is responsible for more than

Correspondence to: Dr Matthias Ocker, Department of Medicine 1, University Hospital Erlangen, Ulmenweg 18, D-91054 Erlangen, Germany

E-mail: matthias.ocker@uk-erlangen.de

${ }^{*}$ Contributed equally

Key words: hepatocellular carcinoma, epidermal growth factor receptor, vascular endothelial growth factor receptor, receptor tyrosine kinase inhibitor, AEE788
600,000 deaths annually (1). Though many local ablative approaches are developed for HCC therapy, the total prognosis of HCC patients is still poor due to the marked resistance to chemotherapies (2).

Recently, the development of receptor tyrosine kinase inhibitors (RTKi) targeting the epidermal growth factor receptor (EGFR) or the vascular endothelial growth factor receptor (VEGFR) has demonstrated good success rates for various human cancers, e.g., lung or colorectal cancer $(3,4)$. EGFR (also designated as ErbB1 or Her1) is a transmembrane receptor tyrosine kinase which has been shown to be overexpressed and to correlate with disease staging, progression and metastasis in HCC (5). As HCC usually presents as a highly vascularized tumor, overexpression of VEGF and its cognate receptors flt-1 (VEGFR1) and flk-1 (KDR, VEGFR2) have been demonstrated previously (6-8). Also here, the expression was correlated with tumor stage, differentiation, progression and overall survival. Interestingly, both EGFR and VEGFR mediate their growth promoting properties via activation of the MAPK pathway, especially the extracellular signal-regulated kinase ERK1/2 (9-11) and a crosstalk and interdependency between these pathways has been described (12).

Previous studies using single EGFR or VEGFR antagonists (e.g., cetuximab (13), gefitinib $(14,15)$, bevacizumab (16) and others) have shown promising anti-tumoral effects on HCC growth in vitro, in animal models in vivo and also in early clinical trials in humans $(16,17)$.

Recently, several new small molecule kinase inhibitors have been developed that target multiple growth factor related pathways. The multi-targeted kinase inhibitor sorafenib has been shown to inhibit the MEK/ERK pathway in liver cancer models (18) and has already been established as a first-line treatment for patients with advanced HCC, although the overall survival has been improved only marginally (19). 
AEE788 is a novel multi-kinase inhibitor that preferably targets the ErbB and the VEGFR family at nanomolar concentrations (20). Previous studies demonstrated a good in vivo tolerability of AEE788 in mice and an enhanced anti-tumor activity in various cancer models, e.g., colon cancer (21) or pancreatic cancer (22), either as a single agent or combined with established cytotoxic agents. Here, we investigated the effect of AEE788 on human HCC cell lines in vitro and in a subcutaneous xenograft model in nude mice after oral administration in vivo.

\section{Materials and methods}

Cell culture. The human hepatoma cell lines HepG2 (p53 $\left.{ }^{\mathrm{wt}}\right)$ and Hep3B (p53 $3^{-/}$, Hepatitis B virus related) as well as primary human foreskin fibroblasts (HF) were obtained from the German collection of microorganisms and cell cultures (DSMZ, Braunschweig, Germany). HepG2 cells were cultured in RPMI-1640 medium (Biochrom, Berlin, Germany) containing $10 \%$ fetal bovine serum (FBS; Biochrom), penicillin (100 U/l), streptomycin (100 $\mu \mathrm{g} / \mathrm{l}$; Biochrom) and gentamycin (40 mg/l; Biochrom) in humified conditions of $37^{\circ} \mathrm{C}$ and $5 \% \mathrm{CO}_{2}$. Hep3B and HF were cultured in Dulbecco's modified Eagle's medium (DMEM, Biochrom) with the same supplements and conditions as described. HF served as a non-malignant epithelial control cell line as primary human hepatocytes are unstable under cell culture conditions.

For all in vitro experiments, 150,000 cells were seeded to 6-well tissue culture plates (Becton-Dickinson, Heidelberg, Germany) $24 \mathrm{~h}$ before treatment. Cells were treated with AEE788 at 0.1-100 $\mu \mathrm{M}$ dissolved in complete growth medium and analyzed or processed for further experiments after 24-120 h. Growth medium was not changed during the experiments.

AEE788 was provided by Novartis Pharma AG (Basel, Switzerland) and was processed as described previously (20). For in vivo experiments, AEE788 was administered to mice orally by gavage at $50 \mathrm{mg} / \mathrm{kg}$ bodyweight three times per week.

Analysis of cell viability and apoptosis. The number of viable cells was determined by counting the number of viable cells after Trypan blue staining in a Neubauer chamber. Cell numbers were then expressed relative to untreated controls set at 1.0 .

Flow cytometry was employed for the quantification of sub-diploid nuclei in treated cell lines after staining with hypotonic propidium iodide solution as descibeed (23). Analysis of labeled nuclei was performed on a FACSCalibur fluorescence-activated cell sorter (FACS) using CellQuest software (both from Becton-Dickinson).

Analysis of mitochondrial membrane potential $\Delta \Psi_{m}$. Mitochondrial injury was measured by JC-1 (5,5',6,6'-tetrachloro1,1',3,3'- tetraethylbenzimidazolocarbocyanine iodide) staining (Sanova Pharma GmbH, Vienna, Austria) according to the manufacturer's instructions. Analysis was assessed by FACSScan after 24-72-h incubation time as described (23).

Immunofluorescence verification of apoptosis. Apoptosis specific cleavage fragments of cytokeratin 18 were detected with the M30 antibody (CytoDeath, Roche Molecular Biochemicals, Mannheim, Germany). Cells were stained according to the manufacturer's instructions after 24-72 $\mathrm{h}$ of incubation with AEE788. Analysis was performed on a Zeiss Axioplan fluorescence microscope (Carl Zeiss, Göttingen, Germany) with OpenLab software (Improvision, Heidelberg, Germany) as described (23).

Xenograft model of hepatocellular carcinoma. HepG2 $\left(5.0 \times 10^{6}\right)$ cells were injected subcutaneously into the flank of 6-8-week old male NMRI mice (Harlan Winkelmann GmbH, Germany). Eight animals were used for each treatment group. Animals were kept in a light- and temperature-controlled environment and provided with food and water ad libitum. Tumor size was determined daily by measurement using a caliper square. When subcutaneous tumors reached a diameter of $7 \mathrm{~mm}$, oral treatment with AEE788 $(50 \mathrm{mg} / \mathrm{kg})$ or vehicle (NMP/PEG300) was started by gavage every other day for a total of five applications. Animals were sacrificed by cervical dislocation and tumor samples were collected two weeks after initiation of treatment. Tumor and tissue samples were fixed in $10 \%$ phosphate-buffered formalin or snap-frozen in liquid nitrogen.

Alanine amino transferase (ALT) levels were determined from blood samples at the beginning and end of the study using an automated procedure on a Cobas Mira (Roche, Mannheim, Germany). Hep3B cells proved not to be tumorigenic in NMRI mice and were therefore not used for in vivo experiments.

All animals received humane care. The study protocol complied with the institute's guidelines and was approved by the Government of Lower Franconia (Würzburg, Germany, file number 54-2531.31-3/06) before the beginning of the experiments.

Protein extraction and Western blot analysis. Total protein was extracted from cultured cells or snap-frozen tumor specimens by adding the sample buffer (23). Samples were subjected to 6-14\% SDS-PAGE (Invitrogen, Carlsbad, USA), transferred to a nitrocellulose membrane and blocked for $1 \mathrm{~h}$ at room temperature in a TBS buffer containing $0.1 \%$ Tween-20 and 5\% low fat milk powder. Membranes were incubated overnight with primary antibodies (p-VEGFR (Flk-1, Merck Biosciences, USA) 1:200, p-EGFR 1:1000, p-MAPK (Erk1 and Erk2) 1:1000, p-AKT 1:1000, MAPK 1:1000, AKT 1:1000 (Cell Signaling Technology, USA), VEGFR 1:200 (Santa Cruz Biotechnology, USA), EGFR 1:500 (Upstate, USA). Membranes were incubated with a peroxidase coupled secondary antibody (1:2000, Sigma) for $1 \mathrm{~h}$ at room temperature. Reactive bands were detected with the ECL chemiluminescence reagent (Amersham Pharmacia Biotech, Freiburg, Germany) and analyzed using GelScan 5 software (BioSciTec, Frankfurt, Germany). Signals were standardized to ß-actin (1:5000, Sigma) content.

Quantitative real-time PCR. For quantitative real-time PCR, total cellular RNA was extracted by use of peqGOLD RNA Pure (Peqlab, Erlangen, Germany) according to the manufacturer's instructions and reverse transcription (RT) was performed as described previously (24). Sequences of primers (Sigma, Germany) and probes (Roche Molecular Biochemicals) 
Table I. Specific primer oligonucleotides and oligonucleotide probes for qPCR.

\begin{tabular}{llll}
\hline Target gene & \multicolumn{1}{c}{ 5'-primer } & \multicolumn{1}{c}{ Probe } & 3'-primer \\
\hline ALAS & CCC TCT TCA CCC TGG CTA A & CCAGGCTG & AGG CAT GGT TCC CAG AAT C \\
VEGF-A & CTACCTCCACCATGCCAAGT & AGGAGGAG & CACCACTTCGTGATGATTCTG \\
VEGF-C & AAGTCCACAGAAATGCTTGTTAAA & CCACCACC & TCGTACATGGCCGTCTGTAA \\
VEGF D & TGAGTGCAAAGAAAGTCTGGAG & CTGCTGCC & TGGTATGAAAGGGGCATCTG \\
NRP 1 & CACATTTCACAAGAAGATTGTGC & GACCTGGA & CATCAATTTTAATTCTGGGTTCTTT \\
Flt-1 & CCACTCCCTTGAACACGAG & GACCTGGA & CGCCTTACGGAAGCTCTCT \\
Flt-4 & AGACAAGAAAGCGGCTTCAG & TGGCTGTG & TTGGGAGTCAGGGTGTGC \\
EGFR & GATCCAAGCTGTCCCAATG & GGAGAGGA & GGCACAGATGATTTTGGTCA \\
\hline
\end{tabular}

are given in Table I. PCR conditions were $95^{\circ} \mathrm{C}$ for $10 \mathrm{~min}$, 40 cycles of $95^{\circ} \mathrm{C}, 60^{\circ} \mathrm{C}$ for $30 \mathrm{sec}, 72^{\circ} \mathrm{C}$ for $1 \mathrm{sec}$ and cooling to $40^{\circ} \mathrm{C}$ for $30 \mathrm{sec}$. Results were normalized to ALAS mRNA content for each sample.

Immunohistochemistry and TUNEL staining. Tumor tissue was fixed with $10 \%$ phosphate-buffered formalin and embedded in paraffin. Sections $(5 \mu \mathrm{m})$ were cut and stored at room temperature until use. Routine histology (hematoxylin and eosin staining) was performed in order to evaluate basic histomorphological features of the specimens. Sections were dewaxed, rehydrated and processed by microwave heating in citrate buffer ( $\mathrm{pH}$ 6.0). Specimens were incubated with primary antibodies (anti-Ki-67, 1:500; anti-ß-catenin 1:200; both from Dako Germany, Hamburg, Germany; anti-EGFR, 1:20, Novocastra, Newcastle upon Tyne, UK) overnight at $4^{\circ} \mathrm{C}$ and visualized using streptavidin-biotin complex (Biogenex, San Ramon, CA, USA) coupled to alkaline phosphatase and developed using Dako Envision polymer (DakoCytomation Co., Hamburg, Germany) and developed using 3-hydroxy-2-naphtylacide-2,4-dimethylanilide or DAB. Endothelial staining was performed with the antiMECA-32 antibody (1:1), produced in a rat hybridoma cell line (25). Tris-buffered saline ( $\mathrm{pH}$ 7.2) served as negative controls. TUNEL stainings were performed with the In Situ Cell Death Detection Kit (Roche, Mannheim, Germany) according to the manufacturer's instructions. Slides were digitized and analyzed with the ImageAccess Enterprise 5 software (Imagic Bildverarbeitung, Glattbrugg, Switzerland). Quantification (extensity) and semi-quantification (intensity and distribution) were performed in each slide, performed with electronic filtering for respective signals.

Statistical analysis. Statistical analysis was performed using SPSS14.0 for Windows (SPSS Inc., Chicago, IL). Significance was calculated using the t-test for unpaired samples. $\mathrm{P}<0.05$ was regarded as significant.

\section{Results}

AEE788 reduces cell viability and induces apoptosis in HCC cell lines. We chose two human HCC cell lines that are representative for human HCC in regard of their p53 status

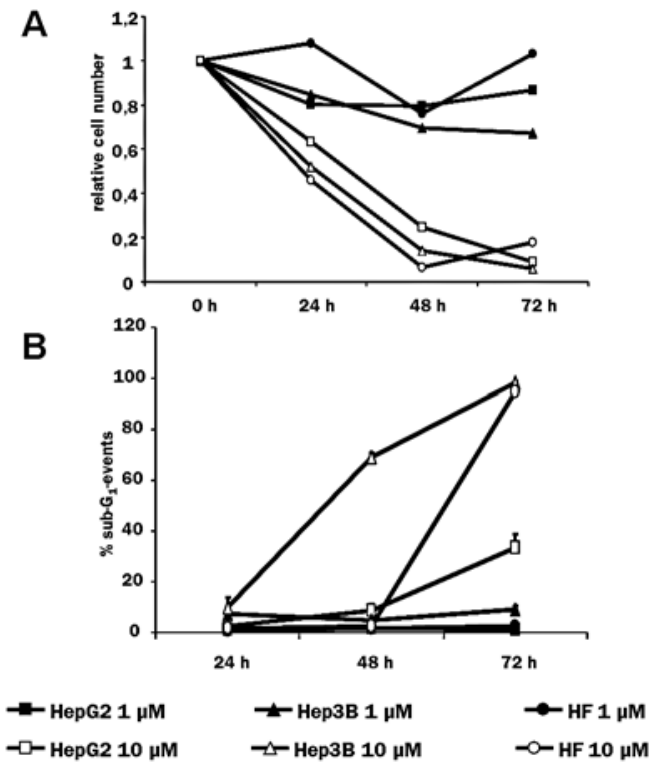

Figure 1. AEE788 reduces cell proliferation and induces apoptosis. (A) Relative number of viable cells after AEE788 treatment was determined after Trypan blue staining in a Neubauer chamber and is expressed relative to untreated controls (1.0). (B) Increase in sub- $\mathrm{G}_{1}$-events as a marker of apoptosis after AEE788 treatment as determined by flow cytometry after propidium iodide staining. Results are mean \pm SEM of three independent experiments.

(HepG2: p53 wild-type, Hep3B: p53 deleted) and viral status (Hep3B: on Hepatitis B virus background). HF cells were used as controls to detect expected skin toxicities in an epithelial but non-transformed cell line.

All investigated cell lines responded to AEE788 treatment with a time- and dose-dependent reduction of the number of viable cells as determined by Trypan blue exclusion test (Fig. 1A). While concentrations $<10 \mu \mathrm{M}$ remained ineffective, concentrations of $10 \mu \mathrm{M}$ or higher lead to a rapid decrease in cell viability. The relative cell number was reduced to $<10 \%$ of untreated controls after 48-72 h. The $\mathrm{IC}_{50}$ value for inhibition of cell proliferation in the investigated cell lines is $\sim 10 \mu \mathrm{M}$.

AEE788 induced apoptosis in the investigated cell lines at concentrations of $10 \mu \mathrm{M}$, while lower concentrations remained ineffective (Fig. 1B). While Hep3B cells showed a significant increase in sub- $\mathrm{G}_{1}$-events already after $48 \mathrm{~h}, \mathrm{HF}$ 

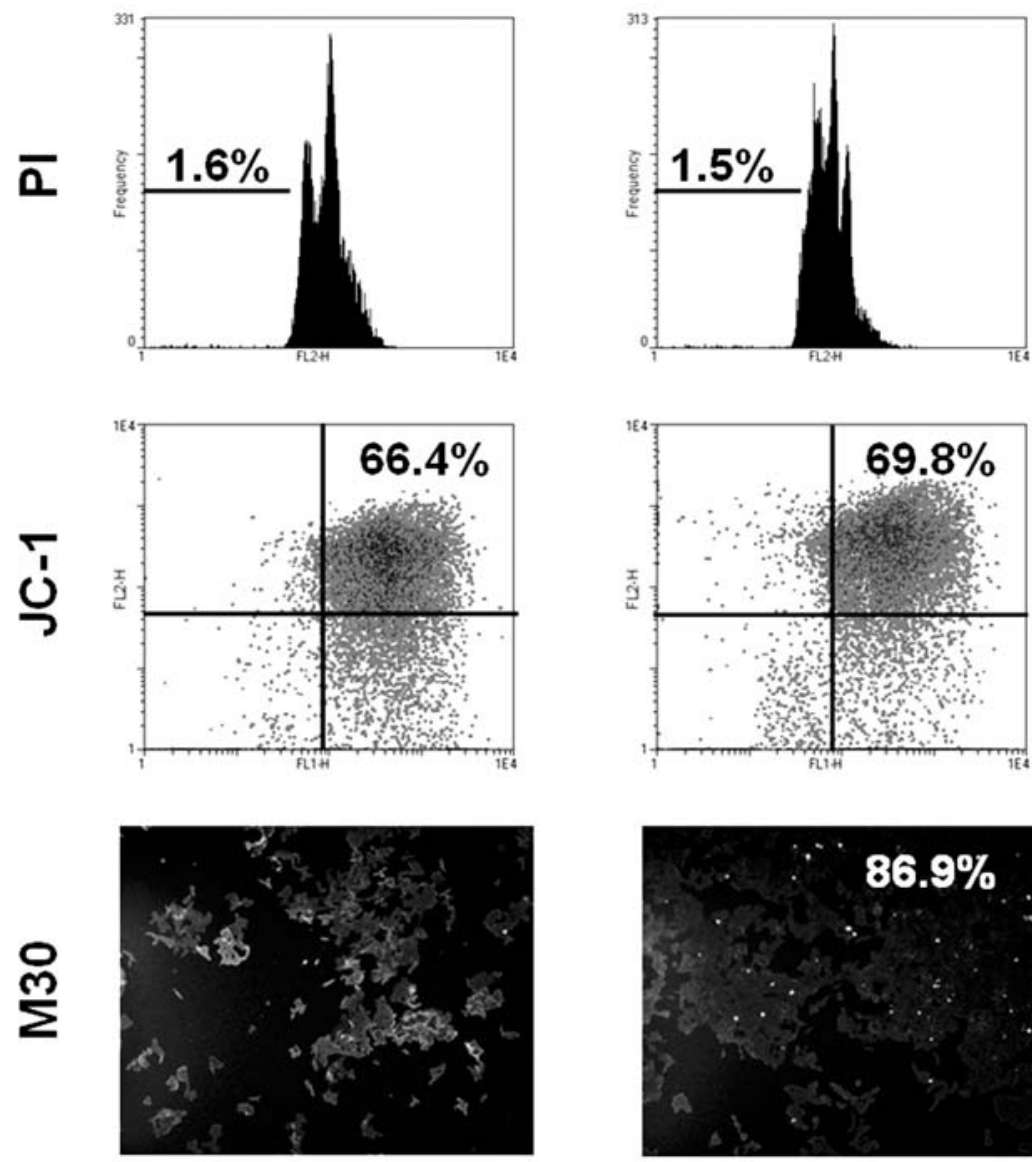

Control

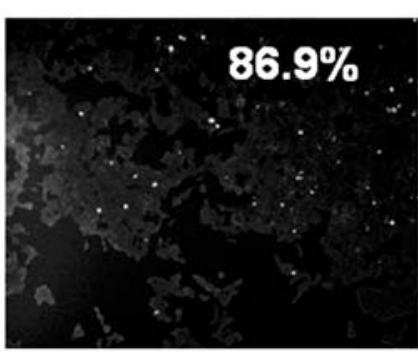

$1 \mu \mathrm{M}$
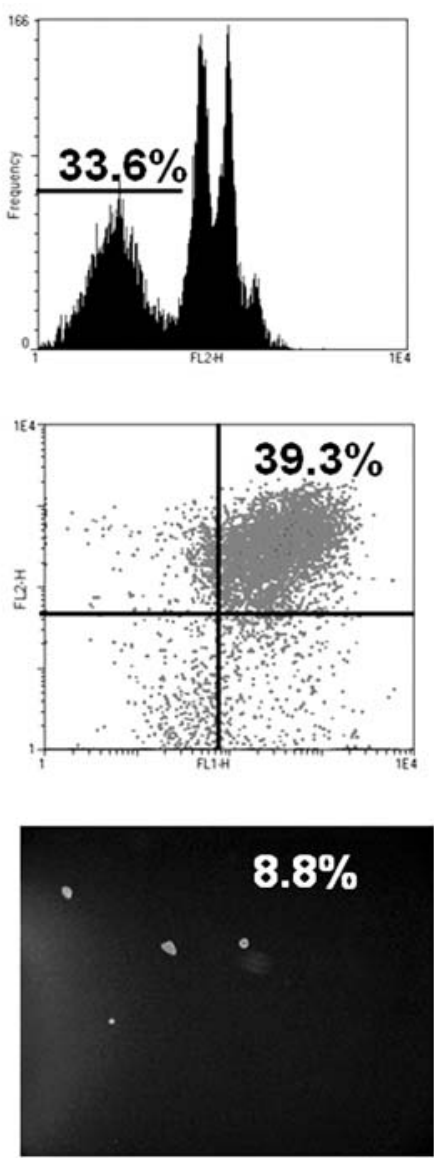

$10 \mu \mathrm{M}$

\section{HepG2}

Figure 2. Mechanisms of AEE788 mediated apoptosis. Shown are examples of HepG2 cells treated with 1 or $10 \mu \mathrm{M}$ AEE788 for $72 \mathrm{~h}$. Top row shows representative flow cytometric histogram analyses of cell cycle and gives the percentage of sub-G $\mathrm{G}_{1}$-events. Middle row shows a representative density plot quadrant analysis after JC-1 staining. X-axis, FL-1H (green fluorescence); y-axis, FL-2H (red fluorescence). Top right quadrant represents cells with intact mitochondria. Bottom row shows immunofluorescence stainings of cytokeratin 18 cleavage fragments using the M30 CytoDeath antibody system. Magnification x200. Percentages indicate cell number relative to untreated controls (100\%) as determined by Trypan blue staining. All values given in this figure are mean values of three independent experiments.

and HepG2 showed a delayed response to AEE788. In HepG2, the maximum apoptosis rate at $10 \mu \mathrm{M}$ reached $58.4 \%$ after $120 \mathrm{~h}$ (not shown).

Mechanisms of apoptosis induction by AEE788. To investigate the mechanisms of apoptosis induction by AEE788 we analyzed its effect on cell cycle, the mitochondrial transmembrane potential $\Delta \Psi_{\mathrm{m}}$ and cytokeratin 18 fragmentation. Representative examples for the following results are shown for HepG2 cells after 72-h treatment with AEE788 in Fig. 2.

Cell cycle analysis by flow cytometry after propidium iodide staining revealed an arrest in $\mathrm{G}_{1}$-phase preceding apoptosis induction. The induction of apoptosis was paralleled by a loss of $\Delta \Psi_{\mathrm{m}}$ as evidenced by a decrease in JC-1 red fluorescence. Interestingly, the more sensitive cell line Hep3B also showed an earlier loss of $\Delta \Psi_{\mathrm{m}}$ compared to $\mathrm{HF}$ and HepG2. In detail, the percentage of cells with intact mitochondria after 72-h treatment with $10 \mu \mathrm{M}$ AEE788 was $0.6 \%$ for Hep3B, $39.3 \%$ for HepG 2 and $3.3 \%$ for HF. These values correlated (Spearman's correlation coefficient $r^{2}$ ) with the apoptosis values determined by flow cytometry with $\mathrm{r}^{2}$ values of 0.99 for Hep3B, 0.98 for HepG2 and 0.99 for HF.

Immunofluorescence staining for apoptosis specific neoepitopes of cytokeratin 18 proved qualitatively that AEE788 mediated cytotoxicity leads to activation of the caspase cascade and not necrotic cell death.

Analysis of EGFR and VEGFR signalling pathways. Semiquantitative Western blotting was used to evaluate the effect of AEE788 on molecules involved in the signal transduction of EGFR and VEGFR (Fig. 3).

We found a constant expression of VEGFR and its phosphorylated active form $\mathrm{p}$-VEGFR in all analysed cell lines which was not affected by AEE788. For EGFR, an expression and phosphorylation was observed in all cell lines. Treatment with $10 \mu \mathrm{M}$ AEE788 lead to a reduction of phosphorylation compared to untreated controls of $76 \%$ and $40 \%$ in Hep3B and HepG2, respectively, and also to a decreased expression 


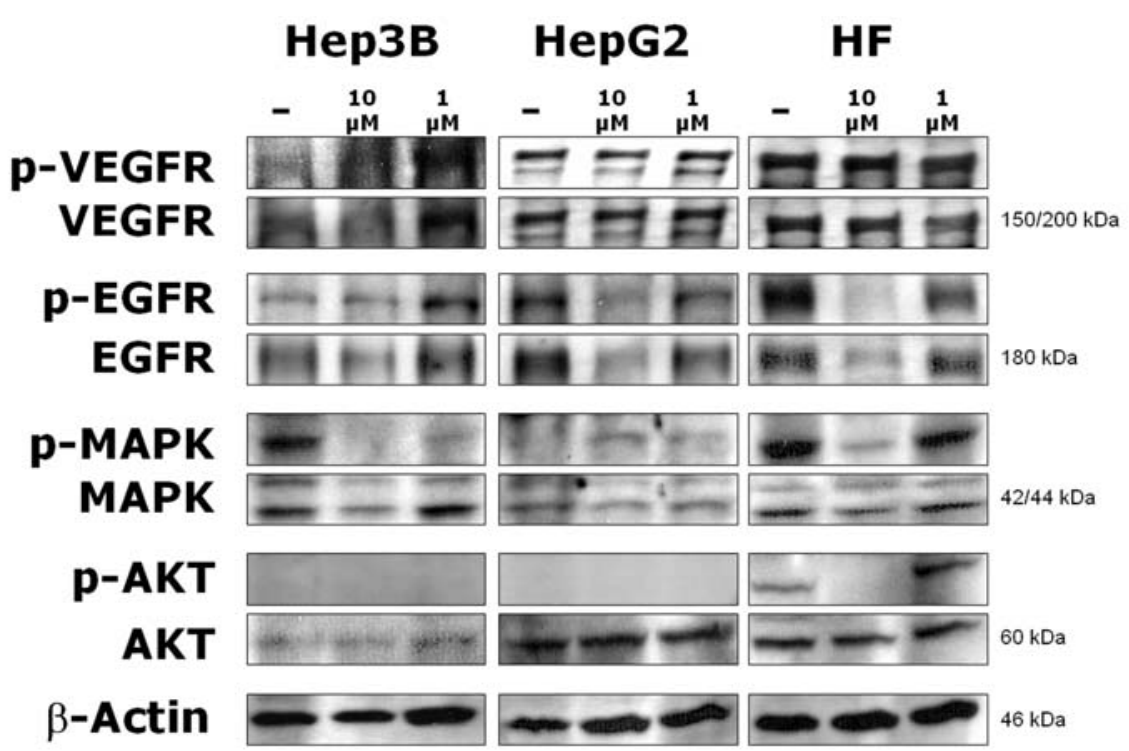

Figure 3. Semiquantitative Western blotting of molecules involved in EGFR and VEGFR signalling. Cells were treated with 1 or $10 \mu \mathrm{M}$ AEE788 for $72 \mathrm{~h}$. Shown are representative examples for three independent experiments for expression of either total or phosphorylated isoforms of VEGFR2 (flk-1), EGFR, MAPK (ERK1/2) and AKT. B-actin was used as a control for equal loading of lanes. Molecular weight of unphosphorylated proteins is given in kDa.

of total EGFR (69\% and 56\% for Hep3B and HepG2, respectively), while $1 \mu \mathrm{M}$ AEE788 did not show these effects.

While the downstream signalling molecule MAPK (ERK1/ 2) was constantly expressed in all three investigated cell lines and no changes were observed upon AEE788 treatment, a strong decrease was detectable for expression of phosphorylated MAPK isoforms. p-MAPK was undetectable after $10 \mu \mathrm{M}$ AEE788 in HF and reduced to $42 \%$ on untreated controls in Hep3B. At $1 \mu \mathrm{M}$, only a slight reduction to 71 and $89 \%$ of untreated controls was observed in HF and Hep3B, respectively. HepG2 cells did not show a reduction of MAPK phosphorylation after AEE788 treatment.

AKT was expressed in all three cell lines. While this signalling molecule was strongly activated in untreated HF cells, p-AKT was not detectable in hepatoma cells and no changes could be observed after AEE788 treatment. In contrast, the activation of p-AKT was strongly suppressed in HF by $10 \mu \mathrm{M}$ AEE788 but remained stable after treatment with $1 \mu \mathrm{M}$.

Quantitative RT-PCR of Hep $3 B, H e p G 2$ and HF cells treated with AEE788. All cell lines showed constant levels of VEGF $\mathrm{A}$ and VEGF D mRNA, while VEGF C was only expressed in HF. In this cell line, AEE788 induced the transcription of VEGF C mRNA about 5- to 9-fold after $24 \mathrm{~h}$. No expression was found for VEGF-R3 in all three cell lines, while Flt1 was highly expressed in HF only. Here, treatment with 1 or $10 \mu \mathrm{M}$ AEE788 lead to a suppression of the cognate mRNA to $15 \%$ of untreated controls after $24 \mathrm{~h}$. The pro-angiogenic co-receptor Nrp1 was expressed in HF and Hep3B but was not affected by AEE788 treatment. EGFR mRNA was detectable in all three investigated cell lines with lowest expression in HepG2 but was not affected by AEE788.

AEE788 inhibits growth of HepG2 xenografts in nude mice. To assess the in vivo effect of AEE788 on the growth of human hepatoma cells, a HepG2 xenograft model was established in male NMRI mice. Animals were treated with
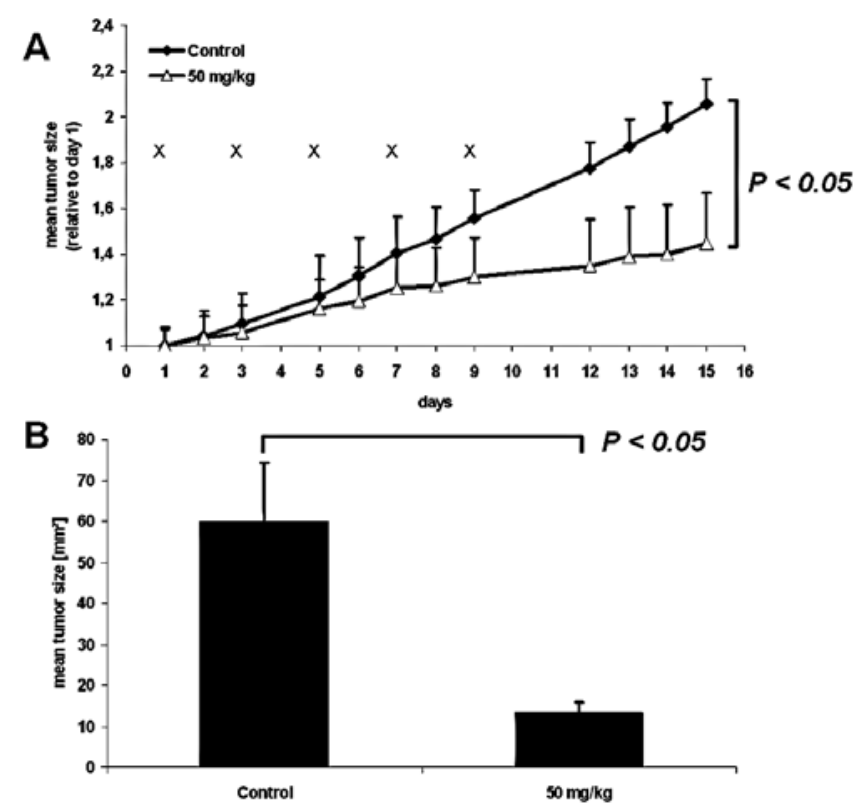

Figure 4. AEE788 inhibits growth of HepG2 xenografts in nude mice. (A) Mice ( $n /$ group $=8$ ) were treated with $50 \mathrm{mg} / \mathrm{kg}$ AEE788 by oral administration three times per week (marked with $\mathrm{x}$ ) for a total of 5 times. Tumor size was determined by measurement with a standardized calliper square and is expressed relative to mean size of day 1 (set at 1.0) \pm SEM. (B) Absolute tumor area was measured from respective histologic cross sections at the study end-point. Results represent mean tumor area in $\mathrm{mm}^{2} \pm \mathrm{SEM}$ from $\mathrm{n}=8$ tumors per group.

oral administration of $50 \mathrm{mg} / \mathrm{kg}$ AEE788 three times per week when tumors reached a diameter of $7 \mathrm{~mm}$ for a total of 5 administrations. From day 7 after initiation of treatment, tumor diameter in the treatment group was significantly smaller than in the control group receiving vehicle only (Fig. 4A). After 2 weeks, the mean relative tumor diameter in the control group was more than doubled compared to day 1 of treatment (206\%) but was increased by only $45 \%$ 
A
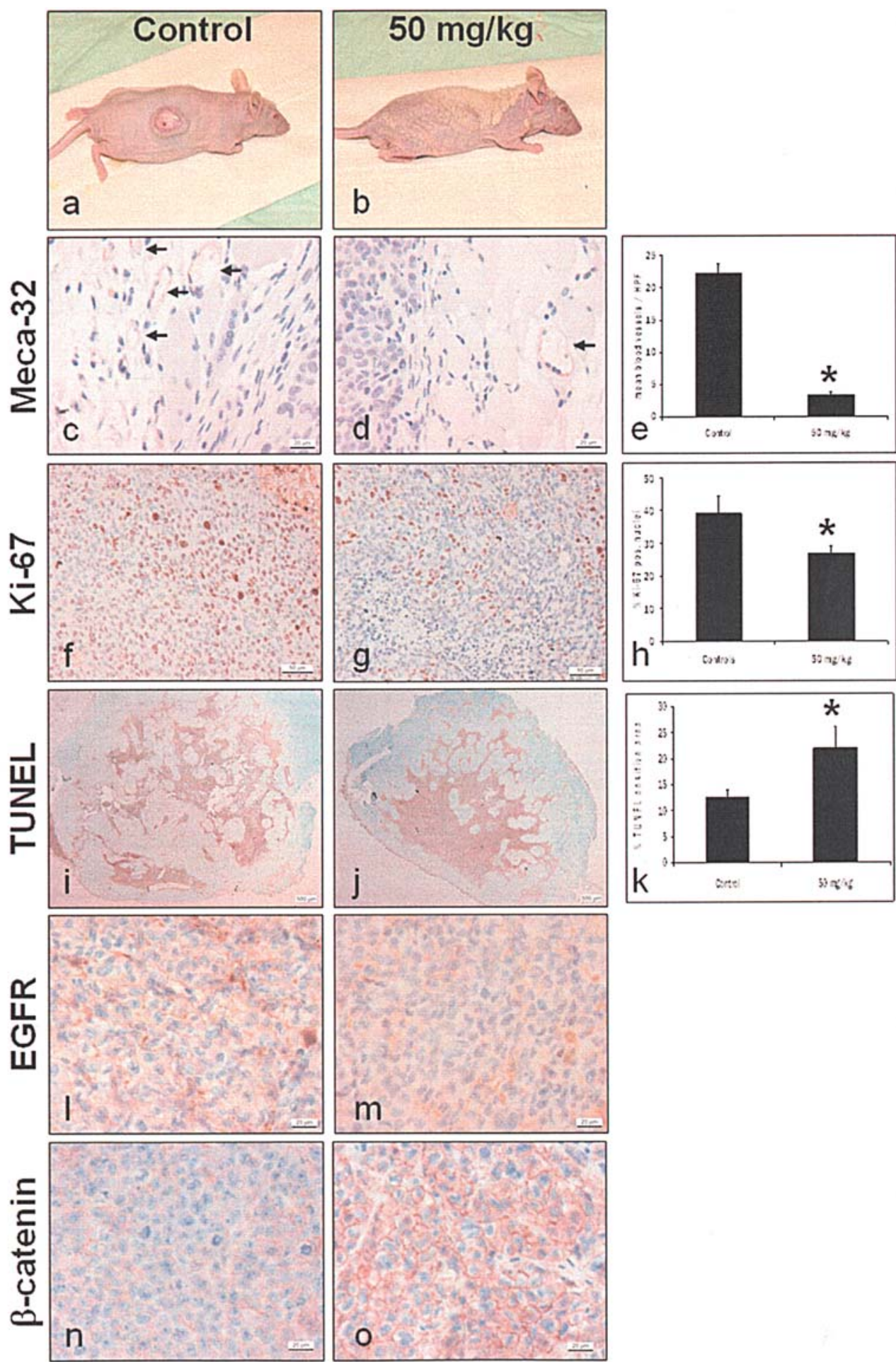

Figure 5. Reduction of tumor growth in nude mice and immunohistochemical analysis. Shown are representative examples of specimens from untreated controls or animals receiving $50 \mathrm{mg} / \mathrm{kg}$ AEE788 by oral administration. (A) Macroscopic image showing a reduced tumor size and a reversible skin reaction in treated mice (a and b). Histochemical sections show endothelial cells (arrows) after Meca-32 staining (c and d, magnification x 400; bar, $20 \mu \mathrm{m}$ ), Ki-67 (f and $\mathrm{g}$, magnification x200; bar, $50 \mu \mathrm{m}$ ), TUNEL stainings ( $\mathrm{i}$ and $\mathrm{j}$, magnification $\mathrm{x} 12.5$; bar, $500 \mu \mathrm{m}$ ), total EGFR expression ( 1 and $\mathrm{m}$, magnification $\mathrm{x} 400$; bar, $20 \mu \mathrm{m}$ ) and localization of $\beta$-catenin ( $\mathrm{n}$ and o, magnification $\mathrm{x} 400$; bar $20 \mu \mathrm{m}$ ). Bar diagrams show quantification of vessel density per group (e), percentage of Ki-67 positive cells (h) and percentage of TUNEL positive area (k). Bars represent mean \pm SEM from eight specimens and 4 independent high power fields per specimen. ${ }^{*} \mathrm{P}<0.05$ vs. control.

in animals receiving $50 \mathrm{mg} / \mathrm{kg}$ AEE788. These results were confirmed by measuring the tumor area at microscopic sections (Fig. 4B). Here, tumors from the control group covered a mean area of $59.6 \mathrm{~mm}^{2}$, while $50 \mathrm{mg} / \mathrm{kg}$ AEE788 reduced the tumor size to $13.2 \mathrm{~mm}^{2}(\mathrm{P}<0.05)$, respectively.
Growth delay by AEE788 is mediated by reduced proliferation and vascularisation. Fig. 5A shows a representative macroscopic image from an untreated control mouse and an animal receiving $50 \mathrm{mg} / \mathrm{kg}$ AEE788. Here, a marked reduction in tumor size is visible that was further analysed by various immunohistochemical stainings. 
B

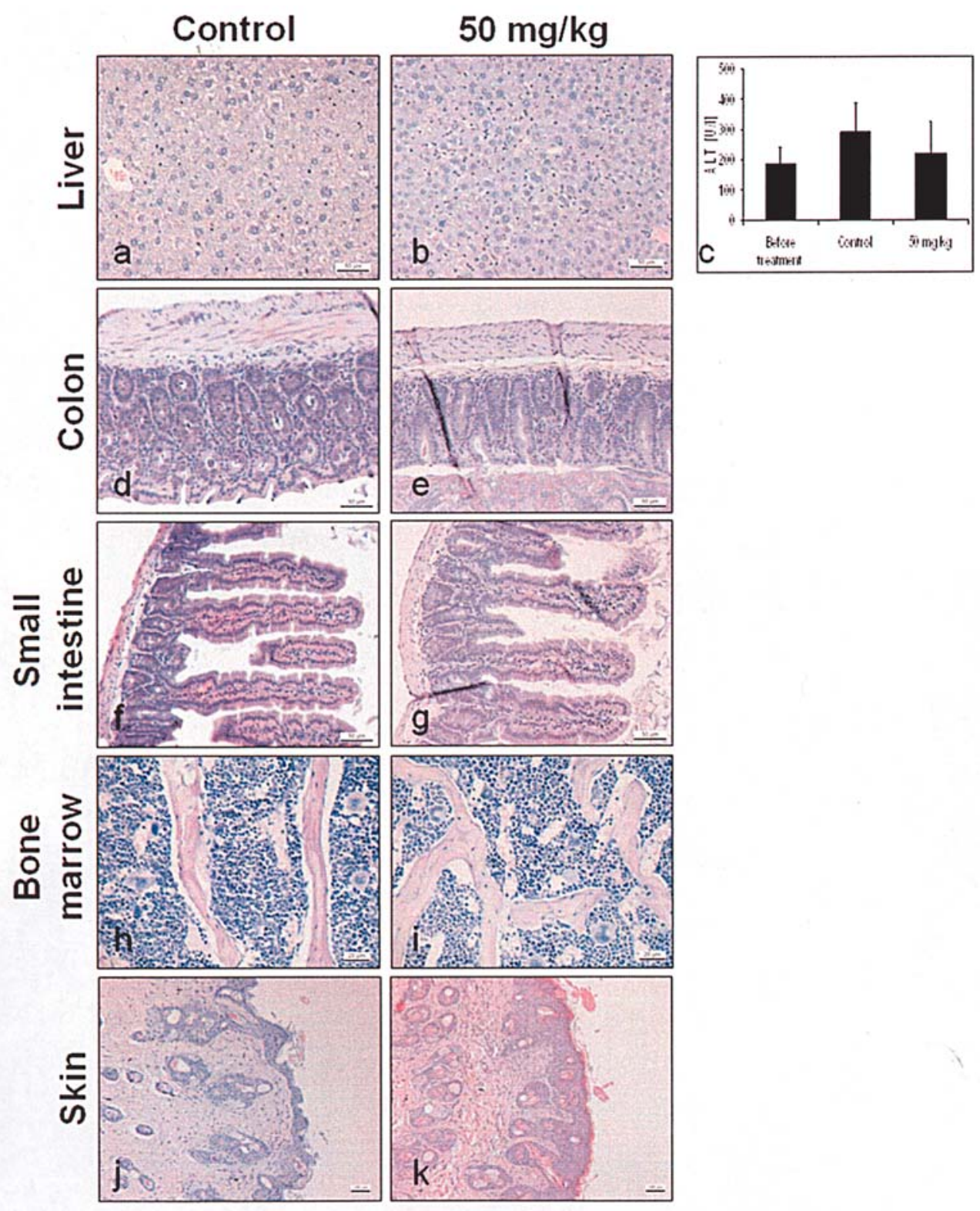

Figure 5 (continued). (B) H\&E stainings of liver (a and b), large (d and e) and small intestine (f and g), bone marrow (h and i) and skin ( $\mathrm{j}$ and k) to assess drug-related side effects. Except for the skin, no morphological signs of toxicity were observed. Magnification x400 (bone marrow); x20 (liver, colon, small intestine); x10 (skin); bar, $100 \mu \mathrm{m}$ (skin), $50 \mu \mathrm{m}$ (liver, colon, small intestine), $20 \mu \mathrm{m}$ (bone marrow). Bar diagram (c) shows serum levels of alanine aminotransferase (ALT) from tumor bearing animals before treatment or receiving either vehicle or AEE788 at $50 \mathrm{mg} / \mathrm{kg}$. Bars represent mean \pm SEM from four animals per group.

The Meca-32 staining revealed a significantly reduced vascularisation of AEE788 treated tumors, i.e. a reduced number and size of blood vessels. The mean blood vessel number per high power field decreased from 22.2 in untreated controls to 3.4 in animals receiving $50 \mathrm{mg} / \mathrm{kg}$ AEE788 $(\mathrm{P}<0.05)$.

A significant reduction in tumor cell proliferation was observed by evaluating the percentage of $\mathrm{Ki}-67$ positive nuclei which was reduced from 39.2 to $26.8 \%$. This was paralleled by a down-regulation of EGFR and a stabilization of $\beta$-catenin in the cell membrane. These results indicate a long-lasting anti-tumor effect of AEE788 as the immunohistochemical analysis was performed 7 days after the last administration of AEE788. In addition, the mean TUNEL positive area increased from 12.6 in untreated to controls to
$21.8 \%$ in tumors treated with $50 \mathrm{mg} / \mathrm{kg}$ AEE788, respectively $(\mathrm{P}<0.05)$.

AEE788 induced a reversible skin reaction in mice. As shown in Fig. 5A, treated mice developed an eczematous skin reaction as has been described for other EGFR inhibitors (26). H\&E staining (Fig. 5B) revealed a desquamation of the epidermis with thickening of the stratum corneum with abundant eosinophilic keratinous material but without additional changes in deeper layers of the skin. Importantly, this skin reaction was completely reversible when administration of AEE788 was stopped. In parallel to this skin reaction, a weight loss was observed in animals treated with $50 \mathrm{mg} / \mathrm{kg}$ AEE788 but not in the other group. Although this weight loss was pronounced $(>20 \%)$ in some animals, all animals 


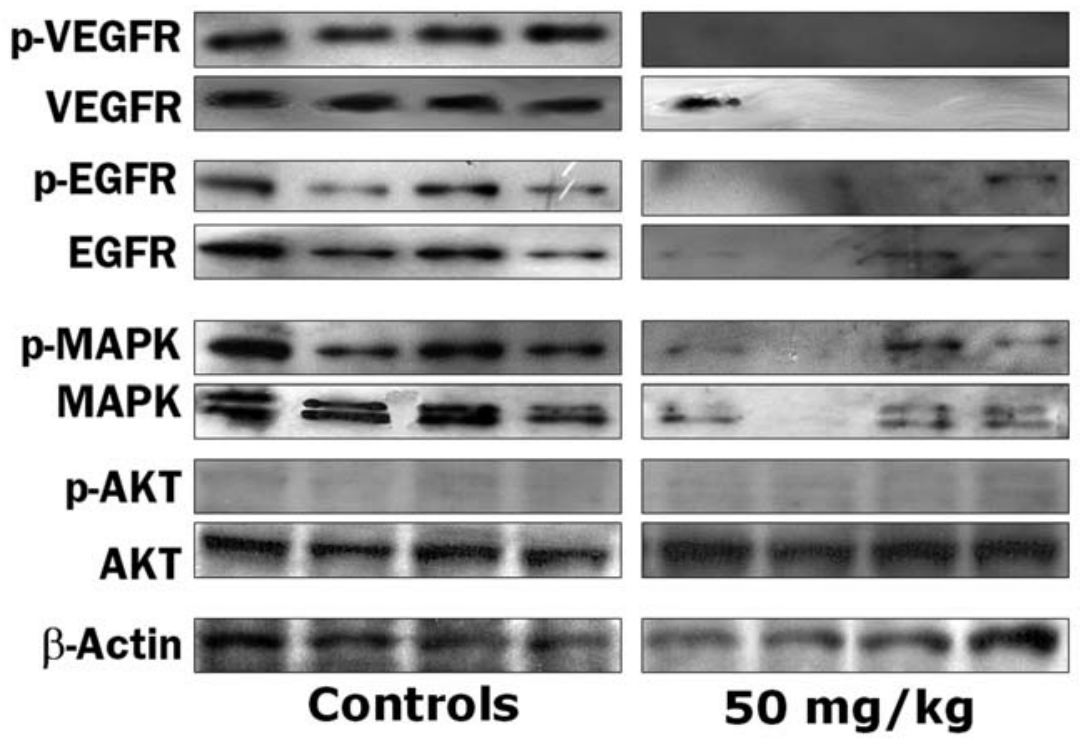

Figure 6. Western blot analysis of representative HepG2 xenograft specimens from four untreated controls or four animals receiving 50 mg/kg AEE788 Tumor samples were obtained at the end of the treatment period and subjected to semiquantitative Western blotting against total and phosphorylated isoforms of VEGFR (Flk-1), EGFR, MAPK and AKT. ß-actin served as an internal control to show equal loading of lanes.

completely recovered after AEE788 was stopped and all animals survived until the study end-point (data not shown). Interestingly, skin reaction and weight loss correlated with tumor response to AEE788.

To determine other putative side effects of AEE788 we investigated further organs by H\&E staining (Fig. 5B). In the liver, only minor signs of a mild drug-induced hepatitis were visible. To assess the degree of liver damage, we determined serum levels of alanine amino transferase (ALT) in tumor bearing mice before and during treatment (Fig. 5B, c). Although ALT levels were elevated also in untreated animals due to the fact that large tumors were established, no statistically significant changes were observed after administration of vehicle control or AEE788 at $50 \mathrm{mg} / \mathrm{kg}$. Other gastrointestinal organs (colon, small intestine) and bone marrow did not show any morphological signs of toxicity due to EGFR inhibition.

AEE788 inhibits activation of MAPK in vivo. As for the in vitro experiments, we also determined the expression and activation levels of EGFR, VEGFR and its downstream signalling pathways by semiquantitative Western blotting (Fig. 6). Untreated HepG2 xenografts showed a strong expression and activation of both EGFR and VEGFR. Treatment with AEE788 lead to a significant reduction of receptor expression which was paralleled by a loss of phosphorylation in these receptors. In parallel to cell culture results, AEE788 also inhibited the activation of the MAPK pathway in the xenografts. Although AKT was also strongly expressed in vivo, no activation was visible in controls or treated mice.

\section{Discussion}

Our results provide evidence that AEE788 inhibits growth of two representative human $\mathrm{HCC}$ cell lines in vitro and in a subcutaneous xenograft model in nude mice after oral administration. This growth inhibition is mediated by an inhibition of activation of the MAPK signalling pathway, pro-apoptotic effects via disruption of the mitochondrial transmembrane potential $\Delta \Psi_{\mathrm{m}}$ as well as an inhibition of tumor vascularisation and tumor cell proliferation in vivo.

AEE788 has been established as a dual receptor tyrosine kinase inhibitor of the EGFR and the VEGFR (20). Both of these growth factor receptors lead to an activation of the MAPK (ERK1/2) pathway (27) which we have shown to be inhibited upon AEE788 treatment by inhibition of receptor phosphorylation. In contrast to previous reports, we did not stimulate cells with recombinant EGF or VEGF and also kept the cells constantly incubated with complete growth medium to see an activation of these pathways as this resembles the actual clinical situation more closely, which explains the rather high concentrations needed for the observed in vitro effects. Yet, also in this setting an inhibition of the MAPK survival pathway was observed and immunohistochemistry revealed a loss of membraneous expression of EGFR in vivo. This receptor has been shown to be regulated post-transcriptionally, e.g., by ubiquitination and subsequent degradation, especially after anti-EGFR therapies (28-30). The posttranscriptional modification of EGFR is in concordance with our findings of reduced total EGFR protein but stable mRNA levels in vitro. The stability of VEGFR expression may be due to the lower affinity of AEE788 to this receptor compared to EGFR (20,31).

$\mathrm{HCC}$ is a highly vascularised tumor and several antiangiogenic strategies have been applied so far (32) and a high microvessel density has been related to poor prognosis in HCC (33). AEE788 has been shown to inhibit proliferation and to induce apoptosis in endothelial cells $(22,34-37)$. Despite the constant growth factor stimulation in vitro and the presence of murine pro-angiogenic factors in vivo, a longlasting reduction of number and size of tumor-associated (murine) blood vessels was observed, paralleled by an 
inhibition the VEGFR pathway but not of the pro-angiogenic co-receptor Nrp1. This indicates a strong anti-angiogenic effect of AEE788 in HCC models in addition to the direct post-transcriptional anti-EGFR effects (e.g., reduced Ki-67 index, loss of survival pathways, disruption of $\Delta \Psi_{\mathrm{m}}$ ) in the hepatoma cells.

Another direct effect of AEE788 on hepatoma cells was the stabilization of $B$-catenin in the cell membrane. B-catenin is a key regulator of gene transcription and cell adhesion (38). Dysregulation or mutation of $\beta$-catenin are considered important steps in the development and progression of human malignancies, including HCC. Activation of the Wnt/ß-catenin pathway is found in up to $67 \%$ of HCC but only $20-30 \%$ exhibit a mutation of these genes, indicating changes in other com-ponents of this pathway (39). Recently, crosstalk between EGFR and B-catenin has been observed in HCC (40) and several kinases, e.g., AKT or the casein kinase family, have been associated with $B$-catenin shuttling to the nucleus $(41,42)$. Our results indicate that AEE788 may interfere with the $\beta$-catenin signalling by inhibition of upstream signals from the EGFR or by inhibition of other intracellular kinases that might have direct action on B-catenin trafficking.

AEE788 was well tolerated in our experiments. Except for the reversible skin reaction and transient weight loss, no severe adverse side effects were observed. Although increased liver toxicity was expected from the known pharmacokinetic data (20), we did not observe any signs of liver damage as shown by ALT levels comparable to controls and normal liver histology. The transient weight loss has not been reported so far from other in vivo studies with AEE788 $(21,22,43)$ and may be due to the different mouse strain used in our experiments but is not a direct effect of AEE788 on intestinal mucosa as evidenced by normal histology. The observed skin reaction correlated with the tumor response to AEE788 treatment and is similar to the commonly observed cutaneous toxicities of other EGFR antagonists (26).

In conclusion, AEE788 represents a novel small molecule inhibitor of the kinase-activity of EGFR and VEGFR and is effective as a single agent in HCC models in vitro and in vivo. Due to its good in vivo properties, the compound could contribute to treatment of human $\mathrm{HCC}$, especially in patients with acquired resistances to present kinase inhibitors or receptor antagonists.

\section{Acknowledgements}

We are indebted to Gabriele Krumholz for support in animal care and experiments. The excellent technical assistance of Ursula Becker, Ines Grob-Achleitner and Astrid Taut is gratefully acknowledged. The study was supported by a grant from the ELAN-Foundation of the University Hospital Erlangen (07.09.24.1).

\section{References}

1. Bosch FX, Ribes J, Diaz M and Cleries R: Primary liver cancer: worldwide incidence and trends. Gastroenterology 127: S5-S16, 2004.

2. Llovet JM, Burroughs A and Bruix J: Hepatocellular carcinoma. Lancet 362: 1907-1917, 2003.

3. Diaz-Rubio E: Vascular endothelial growth factor inhibitors in colon cancer. Adv Exp Med Biol 587: 251-275, 2006.
4. Feld R, Sridhar SS, Shepherd FA, Mackay JA and Evans WK: Use of the epidermal growth factor receptor inhibitors gefitinib and erlotinib in the treatment of non-small cell lung cancer: a systematic review. J Thorac Oncol 1: 367-376, 2006.

5. Ito Y, Takeda T, Sakon M, et al: Expression and clinical significance of erb-B receptor family in hepatocellular carcinoma. Br J Cancer 84: 1377-1383, 2001.

6. Kanno S, Oda N, Abe M, et al: Roles of two VEGF receptors, Flt-1 and KDR, in the signal transduction of VEGF effects in human vascular endothelial cells. Oncogene 19: 2138-2146, 2000.

7. Amaoka N, Saio M, Nonaka K, et al: Expression of vascular endothelial growth factor receptors is closely related to the histological grade of hepatocellular carcinoma. Oncol Rep 16: 3-10, 2006.

8. Yamaguchi R, Yano H, Iemura A, Ogasawara S, Haramaki M and Kojiro M: Expression of vascular endothelial growth factor in human hepatocellular carcinoma. Hepatology 28: 68-77, 1998.

9. Ueda S, Basaki Y, Yoshie M, et al: PTEN/Akt signaling through epidermal growth factor receptor is prerequisite for angiogenesis by hepatocellular carcinoma cells that is susceptible to inhibition by gefitinib. Cancer Res 66: 5346-5353, 2006.

10. Roberts PJ and Der CJ: Targeting the Raf-MEK-ERK mitogenactivated protein kinase cascade for the treatment of cancer. Oncogene 26: 3291-3310, 2007.

11. Huether A, Hopfner M, Sutter AP, Baradari V, Schuppan D and Scherubl H: Signaling pathways involved in the inhibition of epidermal growth factor receptor by erlotinib in hepatocellular cancer. World J Gastroenterol 12: 5160-5167, 2006.

12. Van Cruijsen H, Giaccone G and Hoekman K: Epidermal growth factor receptor and angiogenesis: oportunities for combined anticancer strategies. Int J Cancer 117: 883-888, 2005.

13. Huether A, Hopfner M, Baradari V, Schuppan D and Scherubl H: EGFR blockade by cetuximab alone or as combination therapy for growth control of hepatocellular cancer. Biochem Pharmacol 70: 1568-1578, 2005.

14. Okano J, Matsumoto K, Nagahara T and Murawaki Y: Gefitinib and the modulation of the signaling pathways downstream of epidermal growth factor receptor in human liver cancer cells. J Gastroenterol 41: 166-176, 2006.

15. Hopfner M, Sutter AP, Huether A, Schuppan D, Zeitz M and Scherubl H: Targeting the epidermal growth factor receptor by gefitinib for treatment of hepatocellular carcinoma. J Hepatol 41: 1008-1016, 2004.

16. Zhu AX, Blaszkowsky LS, Ryan DP, et al: Phase II study of gemcitabine and oxaliplatin in combination with bevacizumab in patients with advanced hepatocellular carcinoma. J Clin Oncol 24: 1898-1903, 2006.

17. Philip PA, Mahoney MR, Allmer C, et al: Phase II study of Erlotinib (OSI-774) in patients with advanced hepatocellular cancer. J Clin Oncol 23: 6657-6663, 2005.

18. Liu L, Cao Y, Chen C, et al: Sorafenib blocks the RAF/MEK/ ERK pathway, inhibits tumor angiogenesis, and induces tumor cell apoptosis in hepatocellular carcinoma model PLC/PRF/5. Cancer Res 66: 11851-11858, 2006.

19. Abou-Alfa GK, Schwartz L, Ricci S, et al: Phase II study of sorafenib in patients with advanced hepatocellular carcinoma. J Clin Oncol 24: 4293-4300, 2006.

20. Traxler P, Allegrini PR, Brandt R, et al: AEE788: a dual family epidermal growth factor receptor/ErbB2 and vascular endothelial growth factor receptor tyrosine kinase inhibitor with antitumor and antiangiogenic activity. Cancer Res 64: 431-441, 2004.

21. Yokoi K, Thaker PH, Yazici S, et al: Dual inhibition of epidermal growth factor receptor and vascular endothelial growth factor receptor phosphorylation by AEE788 reduces growth and metastasis of human colon carcinoma in an orthotopic nude mouse model. Cancer Res 65: 3716-3725, 2005.

22. Yokoi K, Sasaki T, Bucana CD, et al: Simultaneous inhibition of EGFR, VEGFR, and platelet-derived growth factor receptor signaling combined with gemcitabine produces therapy of human pancreatic carcinoma and prolongs survival in an orthotopic nude mouse model. Cancer Res 65: 10371-10380, 2005.

23. Ocker M, Alajati A, Ganslmayer M, et al: The histone-deacetylase inhibitor SAHA potentiates proapoptotic effects of 5-fluorouracil and irinotecan in hepatoma cells. J Cancer Res Clin Oncol 131: 385-394, 2005. 
24. Ocker M, Neureiter D, Lueders M, et al: Variants of bcl-2 specific siRNA for silencing antiapoptotic bcl-2 in pancreatic cancer. Gut 54: 1298-1308, 2005.

25. Hallmann R, Mayer DN, Berg EL, Broermann R and Butcher EC: Novel mouse endothelial cell surface marker is suppressed during differentiation of the blood brain barrier. Dev Dyn 202: 325-332, 1995.

26. Lacouture ME: Mechanisms of cutaneous toxicities to EGFR inhibitors. Nat Rev Cancer 6: 803-812, 2006.

27. Sebastian S, Settleman J, Reshkin SJ, Azzariti A, Bellizzi A and Paradiso A: The complexity of targeting EGFR signalling in cancer: from expression to turnover. Biochim Biophys Acta 1766: 120-139, 2006.

28. Marmor MD and Yarden Y: Role of protein ubiquitylation in regulating endocytosis of receptor tyrosine kinases. Oncogene 23: 2057-2070, 2004.

29. Katzmann DJ, Odorizzi G and Emr SD: Receptor downregulation and multivesicular-body sorting. Nat Rev Mol Cell Biol 3: 893-905, 2002.

30. Harding $\mathrm{J}$ and Burtness B: Cetuximab: an epidermal growth factor receptor chemeric human-murine monoclonal antibody. Drugs Today 41: 107-127, 2005.

31. Younes MN, Yigitbasi OG, Park YW, et al: Antivascular therapy of human follicular thyroid cancer experimental bone metastasis by blockade of epidermal growth factor receptor and vascular growth factor receptor phosphorylation. Cancer Res 65: 4716-4727, 2005.

32. Pang R and Poon RT: Angiogenesis and antiangiogenic therapy in hepatocellular carcinoma. Cancer Lett 242: 151-167, 2006.

33. Ribatti D, Vacca A, Nico B, Sansonno D and Dammacco F: Angiogenesis and anti-angiogenesis in hepatocellular carcinoma. Cancer Treat Rev 32: 437-444, 2006.

34. Yigitbasi OG, Younes MN, Doan D, et al: Tumor cell and endothelial cell therapy of oral cancer by dual tyrosine kinase receptor blockade. Cancer Res 64: 7977-7984, 2004.
35. Park YW, Younes MN, Jasser SA, et al: AEE788, a dual tyrosine kinase receptor inhibitor, induces endothelial cell apoptosis in human cutaneous squamous cell carcinoma xenografts in nude mice. Clin Cancer Res 11: 1963-1973, 2005.

36. Younes MN, Park YW, Yazici YD, et al: Concomitant inhibition of epidermal growth factor and vascular endothelial growth factor receptor tyrosine kinases reduces growth and metastasis of human salivary adenoid cystic carcinoma in an orthotopic nude mouse model. Mol Cancer Ther 5: 2696-2705, 2006.

37. Younes MN, Yazici YD, Kim S, Jasser SA, El-Naggar AK and Myers JN: Dual epidermal growth factor receptor and vascular endothelial growth factor receptor inhibition with NVP-AEE788 for the treatment of aggressive follicular thyroid cancer. Clin Cancer Res 12: 3425-3434, 2006.

38. Brembeck FH, Rosario M and Birchmeier W: Balancing cell adhesion and Wnt signaling, the key role of beta-catenin. Curr Opin Genet Dev 16: 51-59, 2006.

39. Lee HC, Kim M and Wands JR: Wnt/Frizzled signaling in hepatocellular carcinoma. Front Biosci 11: 1901-1915, 2006.

40. Tan X, Apte U, Micsenyi A, et al: Epidermal growth factor receptor: a novel target of the Wnt/beta-catenin pathway in liver. Gastroenterology 129: 285-302, 2005.

41. Gao Y and Wang HY: Casein kinase 2 is activated and essential for Wnt/beta-catenin signaling. J Biol Chem 281: 18394-18400, 2006.

42. Fang D, Hawke D, Zheng Y, et al: Phosphorylation of betacatenin by AKT promotes beta-catenin transcriptional activity. $\mathrm{J}$ Biol Chem 282: 11221-11229, 2007.

43. Thaker PH, Yazici S, Nilsson MB, et al: Antivascular therapy for orthotopic human ovarian carcinoma through blockade of the vascular endothelial growth factor and epidermal growth factor receptors. Clin Cancer Res 11: 4923-4933, 2005. 